\title{
sur la gestion et les finances publiques En Août 2021
}

\section{BUDGET DE L'ÉTAT ET DES OPÉRATEURS}

\section{Données générales sur les finances publiques}

Selon Patrick ARTUS la France doit se préparer à une grave crise des finances publiques pour 2023

Dans une chronique publiée sur le site internet du journal Le Point le 28 août 2021, l'économiste Patrick ARTUS considère qu'avec un déficit public total de près de $9 \%$ et un déficit public structurel de 6,5\% du PIB en 2021, les finances publiques de la France doivent se préparer au pire.

Pour l'économiste, dans la mesure où la réponse à la crise de la Covid-19 en France a surtout consisté à accroître des dépenses publiques ou à baisser des impôts, le déficit public restera à un niveau élevé même si la France revenait au plein emploi.

À terme, Patrick ARTUS craint que les investisseurs s'inquiètent de ce que le taux d'endettement public de la France ne baisse pas significativement, d'autant plus que selon lui les règles européennes ne permettront certainement pas à la France de conserver indéfiniment un déficit public supérieur à $6 \%$ du PIB.

Pour revenir à un niveau d'endettement acceptable et pour éviter une crise des finances publiques de la France à I'horizon 2023, la seule solution efficace pour l'économiste serait alors de réduire les dépenses publiques non essentielles, non stratégiques. Des alternatives consisteraient également à ce que la BCE n'arrête pas d'acheter les dettes publiques des États, ou qu'une partie croissante des besoins de dépenses publiques soit financée au niveau de l'Europe et non de chaque État...

\author{
Le monde libre du Gouverneur de la \\ Banque de France
}

Dans un discours prononcé le 26 août 2021, François VILLEROY DE GALHAU, actuel Gouverneur de la Banque de France, évoque sa vision du monde libre qu'il conditionne à une capacité de croissance retrouvée.

Le Gouverneur de la Banque de France croit encore à une croissance cumulée de $10 \%$ sur 2021-2022, et ce pour la France comme pour la zone euro. En utilisant l'expression « So far, so good ", François VILLEROY DE GALHAU rejoint Bruno LE MAIRE pour considérer que la fin du « quoi qu'il en coûte » est certainement arrivée.

Le Gouverneur souligne néanmoins trois défis pour l'économie du monde libre, et pour celle de la France :

- Les difficultés d'approvisionnement qui vont pousser l'inflation à court terme un peu au-dessus des prévisions initiales. Mais elles seront très probablement temporaires, comme dans les reprises précédentes ;

- Plus durablement, les difficultés de recrutement. Malgré les chiffres exceptionnels de la création d'emplois depuis un an $(+630000$ pour l'emploi salarié privé), il existe un « limitateur de vitesse de l'économie française », qui s'est trop vite remise en marche après la crise Covid. Pour le Gouverneur, il n'y a donc pas de réformes plus nécessaires que celles visant à augmenter l'offre de travail : par la formation, par la réduction du sous-emploi des jeunes et des seniors, et par un travail rendu plus incitatif.

- Dernier défi, et celui-là est pour l'Europe entière : la crise Covid a une fois de plus creusé un fossé transatlantique, le fossé de la croissance. L'économie américaine accélère grâce au digital, l'économie européenne et française souffre de son excessive concentration sur l'aéronau- 
tique et le tourisme. II est vital que les européens réussissent deux révolutions majeures: la révolution numérique et la révolution écologique. Si la première devait servir à rattraper un retard certain, la seconde pourrait être l'occasion de donner à l'Europe une avance stratégique.

\section{$\pi$ Procédure budgétaire}

\section{La LOLF fête ses 20 ans !}

Dans une tribune publiée le $1^{\text {er }}$ août 2021 dans le journal Le Monde, Alain LAMBERT et Didier MIGAUD dressent un bilan de ce texte 20 ans après sa promulgation.

Bien que la LOLF ait marqué une révolution copernicienne dans la gestion de nos finances publiques, il y a, pour les "pères de la LOLF», de nombreux motifs de déception dans la mise en application de ce texte. Selon eux, la gestion par la performance telle que résultant de la pratique de la LOLF ne permet pas une véritable gestion en fonction des résultats. La responsabilisation des gestionnaires publics apparaît également encore insuffisante, malgré les ambitions très fortes de la LOLF sur ce point.

Les 20 ans de la LOLF sont également l'occasion pour eux de rappeler l'importance de la mise en œuvre d'une stratégie pluriannuelle des finances publiques. Quoi de plus naturel que de se donner une visibilité sur plusieurs années afin d'assurer une gestion performante des recettes et des dépenses ? Cette vision est toutefois imparfaitement finalisée aujourd'hui.

Pour Alain LAMBERT et Didier MIGAUD, la LOLF contient les outils nécessaires au retour à une gestion saine, efficace et efficiente des finances publiques et donc à la capacité de réduire progressivement notre dette. L'association du Parlement à la maîtrise de la dette doit être également repensée. Si la LOLF prévoit une information plus fournie à destination des parlementaires, l'organisation d'un débat parlementaire solennel sur la maîtrise de la dette, son délai de maturité, ses conséquences pour les générations futures, a toute sa légitimité.

Pour finir les auteurs espèrent un avenir plus radieux à leur œuvre : "Tout parent souhaite la réussite de son enfant: que cet anniversaire soit l'occasion de mettre vraiment en œuvre les principes qui ont présidé à sa naissance. II est temps d'adhérer sincèrement à l'esprit de la LOLF et surtout de le mettre en pratique!».

\section{FinANCES LOCALES}

Les finances des régions entre 2015 et 2021

Dans cette étude, la DGCL poursuit son analyse du paysage financier local dans le contexte de sortie de crise. La situation des régions se dégrade mais dans un contexte d'accélération des dépenses d'investissement. En fonctionnement, les dépenses ont augmenté en 2020 de $+3 \%$ alors que dans le même temps les recettes de fonctionnement ont diminué de - 1,6\%. Aussi, inévitablement, l'épargne brute recule fortement de $-21,6 \%$ en 2020. En 2021, le recul serait bien moindre, de l'ordre de $-5,7 \%$.

Malgré tout, la situation financière des régions n'est pas totalement dégradée, la capacité de désendettement passe de 4,3 ans en 2019 à 6 ans en 2021. On peut sans doute s'attendre à un ralentissement des dépenses d'investissement dans les années qui viennent.

Les finances des départements entre 2015 et 2021

Dans cette étude, la DGCL retrace la dégradation de la situation financière des départements depuis 2020 sous l'influence de la crise actuelle.

Contrairement aux régions, la situation des départements se caractérise par une grande diversité. Par exemple, si l'approche nationale démontre une relative stabilité des recettes, lorsqu'on regarde en détail la situation, de nombreux écarts apparaissent entre territoires. Ainsi, vingt départements ont vu leurs recettes diminuer en 2020, tandis que les autres départements ont vu leurs recettes augmenter, dont vingt-cinq de plus de $+2,5 \%$.

\section{FinanCES SOCIALES}

\section{$\pi$ Situation des comptes sociaux et conséquences de la crise sanitaire}

Le ministre de l'économie, des finances et de la relance annonce la fin du « quoi qu'il en coûte "

Le point de presse de Bruno LE MAIRE le 30 août a sonné la fin du « quoi qu'il en coûte» pour le soutien aux entreprises, en annonçant I'extinction du Fonds de solidarité au $30 \mathrm{sep}$ tembre 2021 (sauf dans les DOM), avec une transition d'un mois assurant une indemnisation de $20 \%$ du chiffre d'affaires ( la dégressivité s'était opérée en sifflet à partir du mois de juin de $40 \%$ à $20 \%$ en août). S'y substituera à partir 
du $1^{\text {er }}$ octobre le dispositif de prise en charge partielle des coûts fixes, déjà en vigueur pour les entreprises de plus de $1 \mathrm{M} €$ de chiffre d'affaires et qui sera ouvert à toutes les entreprises des secteurs S1 et S1 bis, c'est-à-dire celles dont l'activité est réduite du fait de restrictions administratives; pour celles-ci, l'État compensera l'excès de charges fixes par rapport aux revenus à hauteur de $90 \%$ pour les entreprises de moins de 50 salariés et de $70 \%$ pour les plus de 50 salariés, l'instruction des dossiers s'avérant toutefois plus complexe que dans le cadre du dispositif forfaitaire du Fonds de solidarité. Enfin des plans d'action spécifiques seront définis pour les secteurs qui connaissent à présent de véritables difficultés structurelles : événementiel, agences de voyage, montagne... Le système des Prêts garantis par l'État fera l'objet d'un examen avec le secteur bancaire et une clause de revoyure pour l'ensemble des aides aux entreprises est prévu pour le 30 novembre. Ces annonces pourraient marquer la fin d'un engagement sans précédent des finances publiques évalué à $230 \mathrm{Md} €$ au 30 juin 2021 (chômage partiel, Fonds de solidarité, report de charges sociales, PGE) par le Comité de suivi et d'évaluation des mesures de soutien aux entreprises face à la crise de la COVID-19, présidé par Benoît CEUUÉ.

\section{त Politique d'allègement des charges/ coût du travail /mesures en faveur de I'emploi}

\section{Les incitations monétaires au travail ont augmenté entre 2014 et 2019}

Une étude récente de l'INSEE, dont Les Échos ont rendu compte au début du mois d'août, révèle que les incitations à la reprise d'activité mises en œuvre depuis 2014 ont profité aux salariés à faibles revenus. En effet, si on considère le taux marginal de prélèvement effectif sur les salaires, qui indique la proportion de revenu supplémentaire qui est captée par les impôts et les cotisations sociales ou par l'effet de la mise sous condition de ressources des prestations, on observe que celui-ci a baissé jusqu'à 1,7 SMIC, avant de remonter à partir de 2,5 SMIC. Cela résulte de la combinaison des différentes mesures $d^{\prime}$ allègement du coût du travail pour les bas salaires (jusqu'à 1,6 SMIC) qui sont intervenues depuis 2014, puis de la transformation du CICE en 2017, et surtout de la création de la prime d'activité qui a fortement soutenu le revenu des ménages payés à faible salaire.
Le bilan des aides à l'emploi en 2019 selon les statistiques de la DARES

La DARES a publié le 24 août le bilan des dépenses en faveur de l'emploi et du marché du travail en 2019. Celles-ci se sont élevées à $144 \mathrm{Md}$ (soit 5,9 points de PIB), en progression de $0,7 \%$ constants par rapport à 2017 . Ces dépenses se décomposent en 4 catégories : 1) les dépenses pour inciter à l'activité (11 Md€), correspondant principalement à la Prime d'activité, en forte augmentation du fait de la revalorisation importante intervenue au 1 er janvier 2019 ; 2) les dépenses de soutien du revenu -indemnisation du chômage, activité partielle, préretraites (45 Md€) en légère baisse sur la période en lien avec l'amélioration continue du marché du travail ; 3) les dépenses d'incitation à l'embauche-allègements de cotisations, aides sectorielles et géographiques (75 Md€) marquées par la forte réduction des cotisations maladie et chômage suite à la transformation du CICE - qui a dû être compensée budgétairement - et la baisse des effectifs de contrats aidés; 4) les dépenses de formation des personnes peu qualifiées (7 Md€) en diminution. S'y ajoutent les dépenses du Service public de l'emploi (6 Md€).

\section{$\lambda$ Retraites}

\section{La réduction des dispositifs de préretraite} se confirme

Les Échos, faisant état des analyses de la Commission des comptes de la Sécurité sociale, mettent en lumière la réduction très importante des dispositifs de préretraite, qui ont connu un très grand succès en France à partir du début des années soixante jusqu'à la fin du siècle, notamment avec l'allocation spéciale du Fonds national de l'emploi » destinée à accompagner les grandes restructurations industrielles. Le coût très élevé de ces dispositifs a conduit à les recentrer sur les demandeurs d'emploi de plus de 55 ans qui, jusqu'en 2011, ont pu percevoir leur indemnité de chômage jusqu'à leur retraite. Ces dispositifs ont fait place à la mesure de départ anticipée pour carrières longues instaurée dans le cadre de la réforme des retraites de 2003, complétée en 2012 par la possibilité de départ anticipé pour les travailleurs handicapés, et qui a concerné jusqu'à un départ sur quatre. Selon l'analyse précitée, le nombre de départs anticipés ne devrait plus représenter que $5 \%$ du total des départs en 2035 du fait de la démographie (allongement de la durée des études) et de la difficulté de satisfaire la condition d'une carrière complète à 60 ans. La ques- 
tion des carrières longues sera sans nul doute au cœur des débats sur la réforme des retraites, si toutefois celle-ci redevient d'actualité.

\section{$\lambda$ Dépenses liées à la famille/Politique du logement}

Un rapport de l'Institut Montaigne dénonce l'inefficacité de la politique du logement

Un récent rapport de I'Institut Montaigne ("faire sauter les verrous du logement ») met en lumière les limites de la politique du logement menée en France depuis 30 ans, caractérisée par un coût croissant pour les finances publiques (41 Md€ en 2013) et des résultats insuffisants du fait d'un déséquilibre majeur entre l'offre et la demande de logements qui a conduit à une multiplication par 2,5 du prix de I'ancien en métropole depuis 1995. Cette situation résulte, selon I'Institut, d'une gouvernance locale déséquilibrée (la commune ne constitue pas l'échelon territorial pertinent pour la mise en œuvre d'une politique d'urbanisme), de rapports bailleurs/locataires déséquilibrés en faveur de ces derniers, de l'accumulation des normes, ainsi que de la confusion dans l'intervention publique, qui obéit souvent à des objectifs contradictoires entre redistribution sociale et soutien au secteur du BTP. Le rapport formule 11 propositions, dont une grande partie vise à assouplir la réglementation sur le marché locatif et les normes de construction, et surtout à repenser l'intervention de l'État : revoir la fiscalité sur la détention et l'occupation de biens immobiliers (révision des valeurs locatives cadastrales et allègement de la fiscalité sur les transactions), limiter le bénéfice des APL pour les étudiants, stabiliser et concentrer les aides en matière de rénovation énergétique (suppression du crédit d'impôt pour la transition énergétique), faciliter la vente de logements HLM.

\section{గ Assurance-chômage}

L'amélioration de la situation de l'emploi permet au gouvernement d'escompter l'entrée en vigueur de la réforme de l'assurance chômage au $1^{\mathrm{er}}$ octobre

Les deux conditions de « retour à meilleur fortune » auxquelles est subordonnée l'entrée en vigueur de la réforme de l'Assurance chômage (voir Repères de juin) - atteinte d'un seuil minimal de déclarations d'embauche et baisse du nombre des demandeurs d'emploi inscrits à
Pôle Emploi - semblent en passe d'être satisfaites : en effet le nombre cumulé sur 4 mois des déclarations d'embauche a dépassé de 100000 l'objectif visé de 2,7 millions et, d'autre part, le nombre des chômeurs inscrits à Pôle emploi a baissé de 172000 en 6 mois. Dans ces conditions, le gouvernement aurait les justifications nécessaires devant le Conseil d'État pour appliquer à partir du $1^{\text {er }}$ octobre sa réforme de l'Assurance chômage, et en particulier les nouvelles modalités de calcul de l'indemnisation, qui sont susceptibles de rapporter le montant d'économies le plus important.

\section{$\pi$ Solidarité/lutte contre la pauvreté}

Le nombre d'allocataires du RSA en baisse au début de 2021

Selon les dernières statistiques de la CNAF, le nombre de foyers bénéficiaires du RSA, qui avait fortement crû jusqu'à septembre 2020, pour dépasser pour la première fois l'effectif de 2 millions, a commencé à diminuer au dernier trimestre 2020, pour retomber à 1,971 millions à fin mars 2021 (soit une baisse de - $3 \%$ par rapport au précédent trimestre). Cette évolution, qui prend en compte l'effet de la prolongation des droits au chômage, est la conséquence de la forte reprise économique intervenue à la fin de l'année dernière.

\section{FISCALITÉ ET PROCÉDURES FISCALES}

\section{$\lambda$ Politique fiscale}

Baisse d'impôts malgré la crise sanitaire, une voie à poursuivre?

François Ecalle pour l'Institut Montaigne a passé en revue les mesures fiscales de la législature qui s'achève (ex. baisse de I'IS, la transformation de I'ISF, transformation du CICE en allègement de cotisations patronales, PFU, suppression de la taxe d'habitation). Si cette étude souligne la pertinence de certaines baisses de la fiscalité, elle pointe également leur coût budgétaire élevé. Cela représente environ $50 \mathrm{Mds} €$ d'allègement de la pression fiscale, dont la moitié sur les particuliers et l'autre sur les entreprises. Cette étude constate que les économies budgétaires, qui devaient accompagner la réduction des prélèvements obligatoires, ne sont pas advenues. En 2020, la France occupait la première place européenne s'agissant du niveau de ses dépenses publiques $(61,8$ points 
de $\mathrm{P}(\mathrm{B})$, ce qui ne devrait pas s'inverser à court terme. Or avec un taux de prélèvements obligatoires de 44,5\% en 2020, la France dispose encore celui parmi les plus élevés du monde.

Vers une réforme de I'IFER sur les antennes mobiles en loi de finances pour 2022?

Dans un rapport demeuré confidentiel, I'Inspection générale des finances (IGF) a relancé le débat autour de la réforme de l'imposition forfaitaire des entreprises de réseaux (IFER) sur les antennes relais de téléphonie mobile. Cette imposition de toute nature a été mise en place il y a une dizaine d'années suite à la réforme de la taxe professionnelle. Elle est recouvrée à destination des collectivités territoriales (blocs communal et départemental). Son produit pourrait tripler pour atteindre presque $600 \mathrm{M} €$ en 2030 contre $200 \mathrm{M} €$ à ce jour (1 684 , prélevé sur chaque équipement installé sur pylône quelle que soit la technologie - 2G, 3G, 4G, $5 \mathrm{G})$. Les opérateurs de télécommunications dénoncent cette imposition qui, selon eux, pénalise ceux qui déploient le plus d'équipements, qu'ils soient récents comme la $5 \mathrm{G}$ ou pas. Inversement, les détracteurs des antennes relais y voient un juste retour vers les collectivités territoriales. Le caractère déséquilibré de la répartition du produit de ce prélèvement entre les communes rurales et les communes plus densément peuplées est aussi pointé du doigt. L'Inspection générale des finances (IGF) propose plusieurs pistes pour simplifier cet impôt. Des discussions pourraient aboutir à une réforme de cet impôt dans le prochain projet de loi de finances pour 2022. Cette nouvelle possible érosion de la fiscalité locale inquiète les associations d'élus locaux après la réforme de la taxe d'habitation et des impôts de production.

\section{入 Procédure fiscale et contrôle fiscal}

Taxation d'office pour défaut de déclaration d'avoirs figurant sur des comptes ou contrats d'assurance-vie à l'étranger : renvoi d'une QPC au Conseil constitutionnel

En application de l'article L. 23 C du LPF, I'administration fiscale peut demander à une personne physique dans le cadre d'un examen de situation fiscale personnelle (ESFP) des informations ou justifications sur l'origine et les modalités d'acquisition des avoirs placés sur leurs comptes ou contrats dissimulés. A défaut de réponse ou en cas de réponse insuffisante à cette demande d'informations ou de justifications, l'administration fiscale peut adresser une mise en demeure d'avoir à compléter sa réponse dans un délai de 30 jours, en précisant le cas échéant les compléments de réponse qu'elle souhaite. Sans réponse ou en présence d'une réponse jugée insatisfaisante par l'administration fiscale, celle-ci met en application les dispositions de l'article 755 du CGI, entraînant la taxation d'office, ce qui génère un contentieux fiscal important car ce texte est appliqué régulièrement depuis la fin de la procédure de régularisation dite des circulaires "Cazeneuve» (Circ. du 21 juin 2013). Dans un premier temps, la Cour de cassation avait refusé de transmettre quatre QPC au Conseil constitutionnel relatives à ce sujet au motif que ces questions n'étaient pas suffisamment sérieuses (Cass. com, 19 déc. 2019, pourvoi n¹9-15.296). Dernièrement, une nouvelle QPC à ce sujet lui a été transmise par le tribunal judiciaire de Melun par un jugement rendu le 18 mai 2021. Après avoir considéré que les dispositions contestées étaient applicables au litige, qu'elles n'ont pas déjà été déclarées conformes à la Constitution dans les motifs et le dispositif d'une décision du Conseil constitutionnel, et estimant que la question posée présentait un caractère sérieux, la Cour de cassation a considéré qu'il y avait lieu de la renvoyer au Conseil constitutionnel (Cass. Com., 7 juillet 2021, pourvoi $\left.n^{\circ} 21-40.009\right)$. Affaire à suivre.

\section{$\pi$ TVA}

Consultation publique des commentaires relatifs aux nouvelles règles de TVA applicables au commerce électronique

La DGFIP soumet à consultation publique du 13 août au 13 octobre 2021 ses commentaires de la transposition du paquet TVA dit " commerce électronique » entrée en vigueur

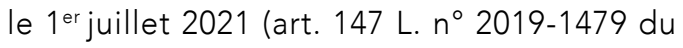
28 déc. 2019 de finances pour 2020 ; v. Repères de l'année 2020). Pour mémoire, l'entrée en vigueur des règles modifiant le régime de TVA du commerce électronique issues du droit de I'UE (mesures adoptées par le Conseil de I'UE en déc. 2017 - «Paquet TVA sur le commerce électronique ») avait été reportée au $1^{\mathrm{er}}$ juillet

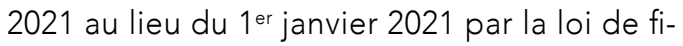
nances pour 2021.

\section{$\pi$ Impôt sur les sociétés}

Un produit symbolique pour la taxe GAFA en 2020

Suite à la publication de la loi de règlement pour 2020 (L. n²021-1039 du 5 août 2021) et à l'occasion de la publication du rapport d'activité de la DGFIP, le produit de la taxe GAFA 
pour 2020 a été dévoilé par l'administration fiscale : $375 \mathrm{M}$, soit près de $100 \mathrm{M} €$ de plus qu'en 2019... Un produit certes limité mais hautement symbolique!

\section{Management public}

\section{$\pi$ Administration numérique}

Bilan d'étape de la conduite des grands projets informatiques de l'État

Depuis 2016, la direction interministérielle du numérique (DINUM) publie trois fois par an son panorama des grands projets informatiques, c'est-à-dire ceux dont le coût est égal ou supérieur à $9 \mathrm{M} €$. L'une des attributions de cette direction est en effet $d^{\prime}$ 'en autoriser le lancement puis d'en suivre le bon déroulement technique, calendaire et financier.

La dernière édition de ce panorama (juillet 2021) qui, désormais, ne paraîtra plus que deux fois par an (contre 3 jusqu'en 2020) dresse un constat plutôt positif. En termes budgétaires, le taux d'écart entre le surcoût effectif et le budget initial s'établit à 19,3\%, pour un coût total de 2,35 Md€. Pour mémoire, en 2020, ce taux se situait à $32 \%$, contre $25 \%$ en 2017. Par ailleurs, l'état des lieux global montre la forte prédominance des projets menés par deux ministères : intérieur (12 projets) et économie et finances (11 projets).

\section{$\lambda$ Fonction publique}

Passe sanitaire et obligation vaccinale dans la fonction publique

Une circulaire du 10 août 2021 précise dans quelle mesure les agents publics sont concernés par le passe sanitaire et l'obligation vaccinale entrés en vigueur le 9 août 2021 après promulgation de la loi sur la gestion de crise sanitaire. Parmi les mesures, on retiendra:

- qu'à partir du 30 août 2021 et au moins jusqu'au 15 novembre 2021, " certains agents publics de l'État seront soumis à l'obligation de présentation du passe sanitaire sur leur lieu de travail»;

- que les « agents publics chargés de missions de contrôle » (ex. police, inspection du travail, services vétérinaires, services de la répression des fraudes, douanes) ne sont pas concernés par cette mesure ;
- qu'il en va de même des agents publics qui interviennent en dehors des horaires d'ouverture des établissements recevant du public et soumis au passe sanitaire ;

- que la vaccination est rendue obligatoire pour I'ensemble des personnels soignants qui ont jusqu'au 15 septembre 2021 pour présenter un schéma vaccinal complet.

\section{EUROPE}

\section{$\pi$ Politique européenne}

\section{Début du versement des fonds NextGe-} neration EU

Au cours du mois d'août 2021, La Commission européenne a commencé à verser à plusieurs États membres un préfinancement correspondant le plus souvent à $13 \%$ des prêts et subventions alloués au pays au titre de la facilité pour la reprise et la résilience : le 3 août, 2,2 Mds€ au Portugal et 770 millions à la Belgique; le 9 août, 4 Mds à la Grèce ; le 13 août, 24,9 Mds à I'Italie ; le 17 août, 9 Mds à l'Espagne; le 19 août, $5,1 \mathrm{Mds}$ à la France et le 26 août, 2,26 Mds à l'Allemagne (9\% de sa dotation).

Aides d'État : autorisation de mesures nationales mais aussi européennes

Après avoir autorisé le 31 juillet 2021 la carte des aides à finalité régionale 2022-2027 pour la République tchèque, au mois d'août, la Commission européenne a continué à autoriser des aides d'État justifiées par la compensation des effets de la crise sanitaire liée à la Covid-19. Elle a ainsi autorisé le 4 août une aide italienne de 430 M€ pour indemniser les exploitants de remontées mécaniques; le 10 août, une aide allemande de $550 \mathrm{M} €$ visant à indemniser Deutsche Bahn; le 27 août, une aide grecque de $500 \mathrm{M€}$ et le mécanisme de capacité de la Belgique pour garantir la sécurité d'approvisionnement en électricité ; le 30 août, une aide italienne de $520 \mathrm{M} €$ pour les foires commerciales. Elle est allée jusqu'à autoriser le 16 août un régime chypriote de $1 \mathrm{Md} €$ et le 27 une aide française de 5,7 Mds destinée à soutenir la production d'électricité par de petites installations solaires sur les bâtiments. Surtout, le 16 août, elle a autorisé un produit de titrisation synthétique dans le cadre du Fonds européen de garantie, géré par le Groupe Banque européenne d'investissement, en vue de renforcer le soutien aux PME dans 22 États membres. 


\section{INTERNATIONAL}

\section{त États}

\section{L'inflation américaine}

Le 11 août, la publication des chiffres de l'inflation américaine montre qu'en juillet, celle-ci reste élevée, mais décélère. Les prix ont augmenté de $5,4 \%$ en juillet sur un an ce qui correspond à une stabilisation au niveau de juin. La Bourse de New York estime que le pire pourrait être passé.

\section{$\pi$ Monnaies}

Cryptomonnaies : le SEC américain veut réguler le "Far West »

Le 3 août 2021, 3 août, le nouveau patron de la Securities and Exchange Commission (SEC, le " gendarme » boursier américain), Gary Gensler a déclaré vouloir réguler les cryptomonnaies en visant en priorité les plates-formes d'échange, et ce, afin de mieux protéger les investisseurs.

Aurélien BAUDU (Fiscalité et procédure fiscale),

Fabrice BIN (Europe, international),

Florent GAULLIER-CAMUS (budget de l'État et des opérateurs),

Matthieu HOUSER (finances locales), Christophe SINNASSAMY (management public),

Yves TERRASSE (finances sociales), Robin DEGRON (coordination).

Retrouvez sur www.rgfp.fr :

- I'intégralité des REPÈRES d'actualité des finances et de la gestion publiques depuis 2015 - le 15 du mois, les REPÈRES d'actualité du mois précédent. 